\title{
Comunicación interna y narrativas transmedia, nuevas estrategias para la empresa responsable. Estudio de caso de Telefónica
}

\author{
Internal communication and transmedia \\ narratives, new strategies for responsible companies. \\ Telefónica case study
}

\author{
Paula Pineda-Martínez; Isabel Ruiz-Mora
}

Cómo citar este artículo:

Pineda-Martínez, Paula; Ruiz-Mora, Isabel (2019). “Comunicación interna y narrativas transmedia, nuevas estrategias para la empresa responsable. Estudio de caso de Telefónica". El profesional de la información, v. 28, n. 5, e280524.

https://doi.org/10.3145/epi.2019.sep.24

Artículo recibido el 10-04-2019

Aceptación definitiva: 27-06-2019

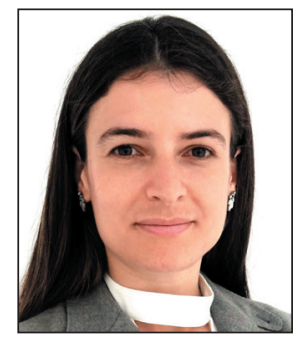
Paula Pineda-Martínez https://orcid.org/0000-0002-2565-1390
Universidad del País Vasco (UPV/EHU) Facultad de Ciencias Sociales y de la Comunicación Departamento de Comunicación Audiovisual y Publicidad
Barrio de Sarriena, $\mathrm{s} / \mathrm{n}$.
48940 Leioa (Bizkaia), España
paula.pineda@ehu.eus

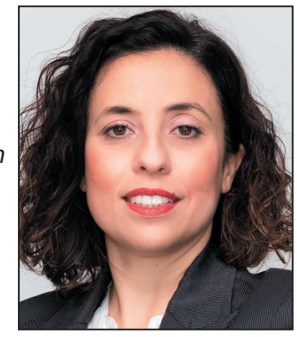

Isabel Ruiz-Mora

http://orcid.org/0000-0003-0723-9850

Universidad de Málaga

Facultad de Ciencias de la Comunicación

Departamento Comunicación Audiovisual y Publicidad

Campus de Teatinos, s/n.

29071 Málaga, España

isabelruiz@uma.es

\section{Resumen}

A partir de la creencia de que la participación y el intercambio constituyen una condición indispensable para una comunicación eficaz con los públicos, este artículo presenta las narrativas transmedia como catalizadores de la comunicación de la responsabilidad social empresarial (RSE) a los públicos internos, a través del caso Play to Move de Telefónica. Este enfoque resulta de especial interés ya que, hasta el momento, la mayoría de las investigaciones en torno a las narrativas transmedia han tratado el tema en relación a contenidos audiovisuales, siendo muy pocos los trabajos que lo han aplicado a la gestión de la comunicación, e inexistentes en lo que se refiere a la comunicación con los públicos internos de manera específica. Con el fin de abordar el tema de estudio, se adopta una perspectiva metodológica cualitativa, basada en una revisión de la bibliografía, y un estudio de caso para ilustrar la aplicación de esas narrativas transmediáticas a la comunicación de la responsabilidad social con los públicos internos. Los resultados demuestran que el transmedia storytelling es un enfoque efectivo de relaciones públicas para comunicar la estrategia de responsabilidad social de las empresas, sin olvidar la importancia de la participación de los empleados en esas políticas responsables. El futuro de las relaciones públicas y de la comunicación interna de la RSE pasa por la confluencia con otras disciplinas como la publicidad o el entretenimiento, y la incorporación a sus estrategias de la gamificación, el storytelling y la transmedialidad.

\section{Palabras clave}

Responsabilidad social empresarial; Negocio responsable; Transmedia; Narrativas transmedia; Gamificación; Comunicación interna; Comunicación organizacional; Comunicación corporativa; Empleados; Públicos internos; Relaciones públicas; Contenido de marca; Película de marca; Interactividad; Yammer; Telefónica.

\section{Agradecimientos}

Las autoras agradecen a Lourdes Tejedor (Dirección de Sostenibilidad y Negocio Responsable en Telefónica) y a Eduardo Puig de la Bellacasa (Dirección Stakeholder Engagement y Reporte Corporativo en Telefónica), por su colaboración y participación en el estudio. 


\begin{abstract}
Based on the belief that participation and mutual exchange are an indispensable condition for effective communication with publics, this article presents transmedia storytelling as a driver of corporate social responsibility (CSR) communication to internal publics, through the Play to Move case study of Telefónica. This approach is of special interest since, to date, most of the research on transmedia storytelling has addressed the subject in relation to audiovisual content, with very few works that have applied it to communication management, and nonexistent in terms of communication with internal publics specifically. In order to address the topic of study, a qualitative methodological perspective is adopted, based on a literature review and a case study to illustrate the application of these transmedia narratives to the communication of social responsibility with internal publics. The results show that transmedia storytelling is an effective public relations approach to communicate the corporate social responsibility strategy, without forgetting the importance of employee participation in these responsible policies. The future of public relations and internal communication of CSR goes through the confluence with other disciplines such as advertising or entertainment, and the incorporation of gamification, storytelling and transmediality into their strategies.
\end{abstract}

\title{
Keywords
}

Corporate social responsibility; Responsible business; Transmedia; Transmedia storytelling; Gamification; Internal communication; Organizational communication; Corporate communication; Employees; Internal publics; Public relations; Branded content; Brand film; Interactivity; Yammer; Telefónica.

\section{Introducción}

La comunicación de la responsabilidad social empresarial (RSE) se encuentra en constante evolución. Torres-Valdés y Campillo-Alhama (2013) señalan que la dirección de comunicación (dircom) debe actuar como un agente de cambio para la generación de nuevos modelos, contenidos, mensajes e información, que construyan credibilidad, apoyo mutuo y confianza. Niño-Benavides y Cortés-Cortés (2018) afirman que los dircoms se enfrentan al reto de vincular la tecnología y sus nuevas vertientes (plataformas, redes sociales, transmedia y multimedia) a toda la acción comunicativa de las empresas, incluida la RSE. Se ha demostrado que la difusión de las tecnologías de la información y la comunicación promueve un nuevo entorno para el desarrollo del diálogo a través de la comunicación de las acciones de responsabilidad social (Ferré-Pavia; Hiyo-Tambra, 2018; Orozco-Toro; Ferré-Pavia, 2013; Capriotti; Moreno, 2007).

Castaño-González (2011) remarca la necesidad de comunicar la RSE no sólo hacia los públicos externos, sino también incluir a los internos como principales stakeholders. Más allá de los réditos económicos que pueden aportar, la autora recuerda que el protagonismo de los empleados en la comunicación de la RSE reforzará

"el sentido de pertenencia, el compromiso, la motivación, la satisfacción en el cargo y el trabajo en equipo, entre otros" (Castaño-González, 2011, p. 173).

Comunicar la RSE contribuye a la mejora de la reputación entre los grupos de interés (Kim, 2011; Orozco-Toro; Ferré-Pavia, 2017), al comunicar las acciones de RSE se fortalece y promueve tanto la identidad como la imagen corporativa (Briceño; Mejías; Moreno, 2010). Por tanto es necesario ver la comunicación no como una herramienta, sino como la estrategia (Ferré-Pavia; Hiyo-Tambra, 2018) que ayudará a conseguir los objetivos perseguidos. Como subrayan Ferrari y Durán (2018), la comunicación es un activo esencial para impulsar los procesos de sostenibilidad. Esto sólo se conseguirá siguiendo una planificación estratégica en la comunicación de la RSE, que gire en torno a los stakeholders como valedores de la propia RSE en la organización y sólo siguiendo un proceso planificado se podrá evaluar el retorno social de lo que se haya invertido en RSE (Sáenz-Acosta; Ventura-Egoávil, 2014).

Al amparo de la RSE se pueden acometer muchos temas de estudio; en nuestro caso abordaremos las relaciones con los públicos internos. Son varios los autores del campo de estudio de las relaciones públicas y la comunicación estratégica que han hecho alusión a este aspecto y a la necesidad de abordarlo como elemento clave (Seitel, 2002; Xifra; Chias, 2008; Castillo-Esparcia, 2009; Míguez-González, 2011; Wilcox; Cameron; Xifra, 2012).

El enfoque perseguido se cimenta en la relación que se establece entre RSE y relaciones públicas en las organizaciones y para ello trabajaremos bajo la definición de RSE del Instituto Ethos de Empresas y Responsabilidad Social:

“Una forma de gestión que se define por la relación ética de la empresa con todos los públicos con los cuales se relaciona y por el establecimiento de metas empresariales compatibles con el desarrollo sostenible de la sociedad, preservando recursos ambientales y culturales para las generaciones futuras, respetando la diversidad y promoviendo la reducción de las desigualdades sociales" (Ethos, 2017, p. 17).

Observamos que es necesario que las organizaciones mantengan una relación con sus públicos basada en el diálogo, teniendo en cuenta sus intereses y demandas y, sobre todo, siendo éticas. Abordar la gestión de la RSE desde las relaciones públicas es ineludible, la gestión de las relaciones con los públicos está tanto en el espíritu de la RSE como en las relaciones públicas (Wang; Chaudhri, 2009, p. 247). 
En este trabajo se estudia una vinculación novedosa de la RSE con la comunicación estratégica transmediática como una nueva forma de transmitir los valores de la RSE y compartirlos con los empleados. Mediante nuevas estrategias comunicativas (narrativas transmedia, storydoing, branded content, entre otras) se implica al empleado en la creación del relato de la RSE y en la transmisión de los valores corporativos asociados al proyecto responsable.

\section{Narrativas transmedia y responsabilidad social empresarial}

Los procesos creativos en la actualidad se encuentran condicionados por la compleja evolución de los medios, soportes y formatos audiovisuales, marcados cada vez más por la existencia de fórmulas de hibridación y mestizaje de contenidos. En el campo de la transcomunicación (Ruiz-Muñoz; Ruiz-Mora, 2018), resultan significativos los casos de nuevas estrategias que combinan relaciones públicas, publicidad, información y entretenimiento para gestionar relaciones y crear experiencias completas.

Al acercarnos al concepto transmedia, debemos detenernos en la definición de Jenkins, precursor del término y el concepto, al definirlo como las historias que son contadas en diferentes medios y que fluyen a través de varias plataformas (Jenkins et al., 2006, p. 46).

La narrativa transmedia se caracteriza por dos aspectos principales:

- una historia que se cuenta a través de múltiples medios y plataformas, donde cada medio constituye un punto de entrada a la historia y realiza su propia contribución a la construcción de la misma;

- la historia puede ser impulsada por las audiencias, ya que una parte de la misma no se limita a consumir el producto cultural, sino que amplía el universo narrativo con nuevas piezas textuales (Scolari, 2013; 2014).

La principal diferencia con el cross-media, término con el que se suele confundir, radica en que en este último el contenido se cuenta a través de diferentes medios y es necesario consumirlos todos para comprender la historia (Davidson, 2008), mientras que la narrativa multiplataforma consiste en la adaptación del mismo relato a varios soportes (Costa-Sánchez; Piñeiro-Otero, 2012).

De hecho, como se ha advertido, las narrativas transmedia cuentan con una serie de especificidades que van más allá de la fragmentación de la historia (Jenkins, 2009):

- Continuidad vs. multiplicidad: continuidad haciendo referencia a la coherencia y credibilidad, y multiplicidad a la posibilidad de acceder a versiones alternativas de los personajes, la historia, el universo...

- Expansión vs. profundidad: la expansión alude a la habilidad del público de difundir contenidos mediante diferentes canales, mientras que la profundidad se refiere al deseo de búsqueda de información.

- Inmersión vs. extracción: la inmersión supone que el espectador se "zambulle" en todos los mundos del universo transmedia, y la extracción, cómo se integran elementos de ese universo en la vida cotidiana.

- Construcción de mundos: son las extensiones de la narrativa y se producen mediante experiencias entre el mundo real y mundo digital, lo que está relacionado con los conceptos de inmersión y extracción previamente citados.

- Serialidad: se lleva a cabo en múltiples entregas.

- Subjetividad: los espectadores exploran la historia gracias a los personajes y sus puntos vista.

- Ejecución: cuando existe la posibilidad de que el trabajo realizado por los fans se convierta en parte de la propia narrativa transmedia (si se logra que el público participe activamente).

En la descripción del funcionamiento del escenario transmedia las historias pueden ser instrumentos para motivar a los públicos internos (La-Roda-Gómez, 2017) y que se conviertan en co-creadores del discurso de la organización, expandiendo los discursos, las historias y los mensajes. Fruto de esta estrategia se obtienen beneficios que van más allá de los resultados económicos,

"la conexión emocional que se establece con el consumidor va mucho más allá del mero acto de consumo y permite mantener una experiencia con la marca que sirve de fundamento para establecer relaciones a medio y largo plazo" (Galán, 2016, p. 922).

Al mismo tiempo, tampoco se reduce a transacciones comerciales y este enfoque innovador (Saavedra-Bautista; Cuervo-Gómez; Mejía-Ortega, 2017) puede ser aplicado para contar historias y generar experiencias con múltiples aplicaciones y escenarios, como puede ser entre otros el caso de:

- marcas de alimentación (Galán, 2016);

- conjuntos arqueológicos como el caso de Atapuerca (Burgos, España) donde se aplica una estrategia transmedia para promocionarlo (Chomón-Serna; Busto-Salinas, 2018);

- promoción de series de televisión (Villén-Higueras, 2017);

- procesos de educación (Robin, 2005; Sugathan; Kalid, 2009; Grandío-Pérez, 2016);

- aprendizaje de idiomas (Tsou; Wang; Tzeng, 2003);

- simulación de inteligencia colectiva para la toma de decisiones (Rodríguez-Ruiz; López-Peinado; González-Gutiérrez, 2015);

- socialización de la adolescencia (Würfel, 2014);

- aprendizaje a través de experiencias transmediáticas (Corona-Rodríguez, 2015; Scolari et al., 2018), entre otros. 
El aspecto común y el éxito de las narrativas transmedia radica en que esta estrategia permite

"fidelizar a los usuarios porque generan experiencias que comparten y que les hacen sentirse partícipes del relato" (Chomón-Serna; Busto-Salinas, 2018, p. 941).

Pero como apunta Scolari

"la participación de los usuarios en la expansión hace imposible saber dónde termina un mundo narrativo transmedia" (Scolari, 2014, p. 73).

Profundizando en el funcionamiento de una estrategia transmedia, encontramos que

"se extienden de un extremo a otro de la ecología mediática, abarcando viejos y nuevos medios. También atraviesan los géneros: hay narrativas transmedia en la ficción, en el periodismo, el documental o la publicidad" (Scolari, 2014, p. 73),

y las relaciones públicas, la educación, la sociabilización..., llegando a la responsabilidad social.

En el ámbito de la responsabilidad social, algunos trabajos se han aproximado a la estrategia transmedia como recurso para alcanzar los fines responsables. Fuentes-Gómez-Calcerrada y González-Martín (2015) recurren a la narrativa transmedia como metodología docente para fomentar el emprendimiento responsable. Como el caso de Chipotle, una marca que promueve el consumo entre productores locales a través de una estrategia transmedia que combina la promoción, un cortometraje y un videojuego para móvil; la estrategia contribuye a mejorar la visibilidad de la marca, el engagement y una práctica comercial responsable (López-Castillo, 2017). Es destacable el trabajo de Rodríguez-Terceño, Caldevilla-Domínguez y Ramos-Fernández (2015) sobre drama management como estrategia transmedia para mejorar la imagen social corporativa, una estrategia que supone el desarrollo de piezas de cine producidas por y para empresas con el fin de mejorar su imagen y transmitir valores (Rodríguez-Terceño, 2014, p. 620). Galbiati y Venditti (2017), tras su investigación en educación y buscando una estrategia para comunicar la RSE de los proyectos de diseño, concluyen que las acciones de storytelling y transmedia en el entorno digital suponen una forma atractiva para establecer una relación entre las empresas y la sociedad, así como para comunicar de forma estratégica la RSE.

El trabajo de Coombs (2019) explora cómo el transmedia es una estrategia que debe ser empleada para comunicar la RSE y analiza el caso de Procter \& Gamble's "Thank you mom". Nicoli y Komodromos (2019) exploran el caso del Banco de Chipre y la estrategia de comunicación seguida por la entidad en el entorno digital, examinando las nuevas narrativas transmedia. Por otro lado, Maltseva, Fieseler y Trittin-Ulbrich (2019) demuestran que la gamificación como estrategia para comunicar temas sociales y medioambientales puede tener resultados ambiguos, pero que con un diseño riguroso de la experiencia que fomente el aprendizaje de los stakeholders puede ser de gran utilidad.

\section{Metodología}

Con el fin de afrontar el tema de estudio, se adopta una perspectiva metodológica cualitativa basada en una revisión de la bibliografía y un estudio de caso. Concretamente se analiza el caso de Play to Move de Telefónica para ilustrar la aplicación de las narrativas transmedia a la comunicación de la responsabilidad social corporativa con los públicos internos. La combinación de técnicas escogida para el análisis de caso considera el análisis documental y la entrevista en profundidad con responsables de la empresa.

El estudio de caso se considera un método apropiado cuando nos enfrentamos a estudios exploratorios en los que los temas que se trabajan son novedosos o no han sido estudiados en profundidad hasta el momento (Yin, 2013). Ha sido aplicado previamente en estudios similares demostrando su utilidad (Costa-Sánchez; Piñeiro-Otero, 2012; Costa-Sánchez, 2013; Mayor-Mayor, 2014).

La selección del caso de estudio se realizó:

- por tratarse de una campaña que incorpora cuatro elementos fundamentales en comunicación en la actualidad: responsabilidad social, gamificación, narrativa y transmedialidad;

- por tratarse de una campaña sin precedentes en relación con el tema de estudio, ya que ha sido considerada como la primera campaña transmedia de comunicación interna de una empresa española (Llorente \& Cuenca, 2017), y ha sido ampliamente galardonada y reconocida como caso de éxito en el entorno profesional (Corporate Excellence, 2018). La campaña permite analizar el tema desde la perspectiva de una empresa con una amplia experiencia en lo que se refiere a la innovación en comunicación.

Las entrevistas en profundidad se llevaron a cabo en febrero de 2019, con una duración aproximada de una hora, y aplicando un guion de preguntas-tema semiestructurado, de manera que se mantuviera el foco de la entrevista permitiendo al mismo tiempo que surgieran perspectivas individuales (Patton, 1990). Las personas entrevistadas, Lourdes Tejedor (Dirección de Sostenibilidad y Negocio Responsable en Telefónica) y Eduardo Puig de la Bellacasa (Dirección Stakeholder Engagement y Reporte Corporativo en Telefónica), fueron seleccionadas por haber participado en la campaña objeto de estudio, así como por ocupar diferentes roles dentro de la cadena de gestión de la comunicación en la compañía.

El estudio analiza en primera instancia los principales componentes de una narrativa transmedia (Scolari, 2016) aplicados a la comunicación interna: 
- la historia,

- los medios y plataformas que se utilizan para expandir la narración, y

- el papel que los públicos han tenido en la creación y desarrollo del relato.

Un segundo análisis examina los efectos de la estrategia transmediática y las posibles limitaciones de la misma como catalizadores de la responsabilidad social entre los públicos internos.

\section{Resultados}

\subsection{Contextualización: objetivos y mensajes}

Play to Move es una campaña internacional desarrollada en 2016 por Telefónica en el marco de su nuevo Plan global de negocio responsable. La iniciativa tenía por objetivo dar a conocer a los empleados los principios de negocio responsable de la compañía (Telefónica, 2016), así como las actuaciones que ésta estaba realizando para llevarlos a cabo. Adicionalmente la iniciativa también pretendía que los empleados sintieran estos principios como algo propio y aplicable en su día a día, de manera que se generara una cultura de responsabilidad entre el público interno.

Estos principios de negocio responsable, consistentes en una serie de pautas sobre las que la compañía rige sus actuaciones para garantizar que éstas sean responsables, resultaban demasiado abstractos, densos y ajenos para los empleados. Esto, unido a un contexto de híper-saturación informativa, llevó a Telefónica a buscar fórmulas diferentes para comunicar internamente lo que la compañía estaba realizando en materia de responsabilidad.

La campaña tuvo una duración de 1 año y se llevó a cabo con la colaboración de la agencia Llorente \& Cuenca y la participación de varias áreas de la compañía: Ética Corporativa y Sostenibilidad, Comunicación Interna y Recursos Humanos, aunque también se contó con el apoyo de otras áreas como la de Negocio, Transformación Digital y Relaciones Internacionales, para validar la información con los responsables y al mismo tiempo buscar aliados en toda la compañía.

\subsection{Estrategia}

La campaña se articuló a través de un eje creativo global (Play to Move) que permitió planificar toda la estrategia de comunicación. Ambas palabras transmitían una llamada a la acción y al disfrute de los empleados tanto hacia la campaña como hacia los principios de negocio responsable de la empresa, y al tratarse de una expresión inglesa, permitía su aplicación a todos los países en los que está presente la compañía.

La campaña utilizó la narrativa, o arte de contar cuentos o historias, para generar un relato lúdico que apelaba a las emociones, daba sentido a la realidad y resultaba altamente didáctico y mnemotécnico para los empleados (Salmon, 2008). Además, la empresa apostó por una campaña transmedia de comunicación interna, de manera que fueran los propios empleados los que co-crearan una narración que se iba construyendo a través de diferentes plataformas y formatos (aplicaciones, redes sociales, brand film...).

https://www.youtube.com/watch?v=AzjcRYogH8s

Durante toda la campaña se utiliza un tono cercano que favorece la identificación de los empleados con la historia, alejándose de un estilo más informativo y lejano al que el público interno estaba acostumbrado hasta el momento. Los valores que se buscaba transmitir están asociados a la excelencia en las conductas responsables, la transparencia, la cotidianeidad y la cercanía. La campaña, a pesar de que pretende transmitir un mensaje de transparencia y responsabilidad, está cargada de un alto corporativismo y ausencia de autocrítica, tal y como puede apreciarse en el film.

\subsection{Medios y formatos}

Teniendo en cuenta el contexto hiperconectado e infoxicado en el que se desarrollaría la campaña, Telefónica optó por utilizar medios y formatos diferentes e innovadores que evitaran los cauces más saturados de la compañía y consiguieran captar el interés de los empleados. Se otorgó un especial protagonismo al digital con el objetivo de alcanzar a una audiencia global de acuerdo con los requerimientos de la campaña.

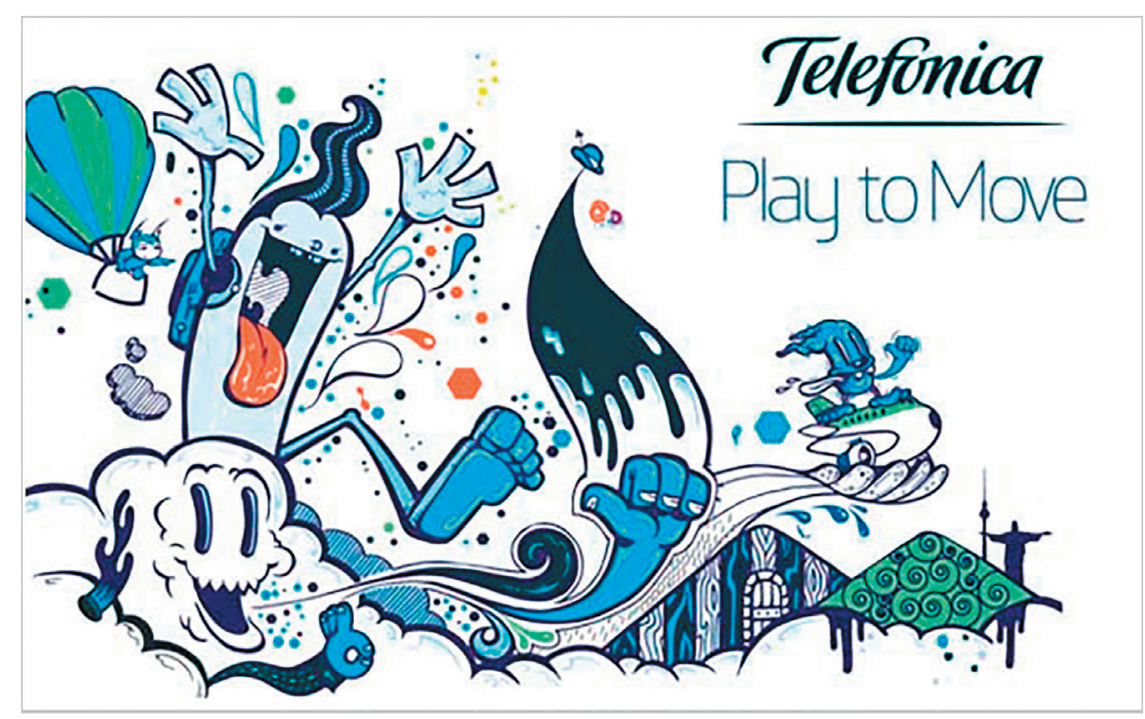

Figura 1. Gráfica Play to Move Fuente: Llorente \& Cuenca (2016) https://informeanual2016./lorenteycuenca.com/wp-content/uploads/2017/02/play2.jpg 
La plataforma central utilizada durante la iniciativa fue Yammer, la red social que utilizan los empleados de Telefónica. https://products.office.com/en-us/yammer/yammer-overview

La principal acción realizada en la misma fue una gamificación multijugador online a la que se accedía a través de la red social. La compañía optó por el uso de la gamificación con el fin de dar a conocer los principios de negocio responsable de una manera cercana y atractiva para el público interno, y generar expectación respecto a la campaña. Mediante el juego los empleados se retaban mutuamente para demostrar quién sabía o descubría más sobre los proyectos de la empresa. Con ello se pretendía que el juego fuera lo suficientemente motivador como para que la mayor cantidad de empleados a nivel global quisiera participar en él de manera activa.

Así mismo, desde el comienzo de la campaña y hasta su finalización se creó un grupo en Yammer destinado a la dinamización de la iniciativa. Así, se consiguió generar una comunidad estable de empleados implicados en la campaña. Para dinamizar el grupo se utilizaron publicaciones de texto e imagen, píldoras audiovisuales, y otros contenidos transmedia que contribuían a expandir la historia.

Como resultado de la gamificación fueron seleccionados 3 empleados para participar en un viaje experiencial a 3 países en los que está presente la compañía (Brasil, España y Perú). Durante el viaje los protagonistas visitan desde un centro de datos de la compañía en Valencia (España) hasta una comunidad indígena en Iquito (Perú), para comprobar de primera mano cómo se materializan los principios de negocio responsable de $\mathrm{Te}$ lefónica en los esos países.

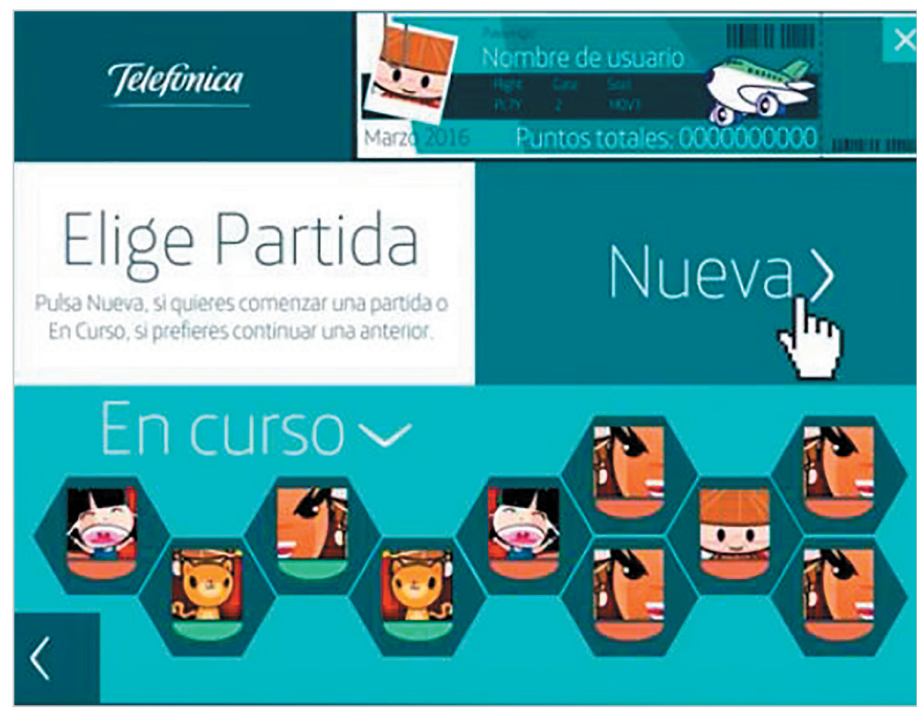

Figura 2. Gamificación Play to Move

Fuente: Telefónica

El resultado del viaje fue un brand film documental, el primero realizado por Telefónica (Telefónica, 2016a y 2016b), en el que los tres empleados narran la historia hablando a la cámara acerca de sus experiencias y reflexiones durante la aventura. El estreno de la película se realizó en Madrid e incluyó un evento en el que se invitó a empleados de la compañía, y se realizaron otras actividades además del visionado de la película (coloquio, show cooking...). También se realizaron acciones para promocionar el estreno, tales como la creación de una identidad visual exclusiva para el evento, Street Marketing en el propio Distrito de Telefónica o formatos de comunicación para su difusión a través de canales internos, entre otros.

El viaje también se transmitió al resto de empleados en el grupo de Yammer en forma de micro-relatos, que contribuían a la expansión y comprensión de la historia, pero al mismo tiempo permitían conectar con el relato sin tener que consumir todas y cada una de sus partes.

Como apoyo a la estrategia se puso en marcha una acción bajo el nombre "Micro-retos Play to Move", en la que se pedía a los empleados que dieran ideas de innovación para promover el impacto positivo de la compañía en la sociedad de acuerdo con los principios de negocio responsa-

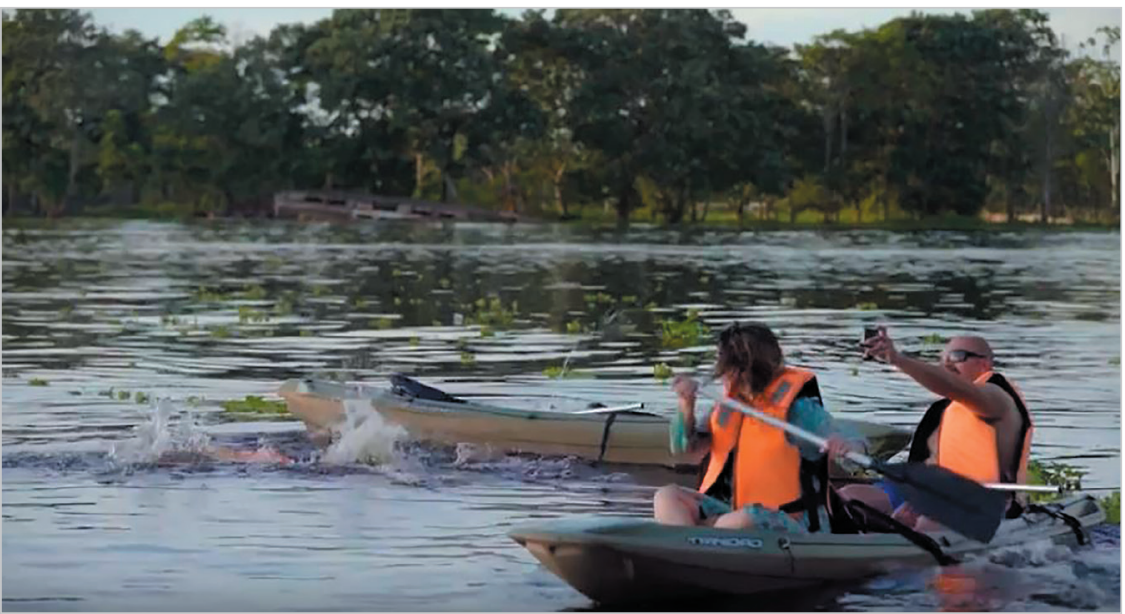

Figura 3. Secuencia del brand film Play to Move https://www.youtube.com/watch?v=AzjcRYogH8s Fuente: Telefónica ble. Finalmente se eligieron tres de las ideas participantes.

Durante todo el proceso se utilizaron como apoyo el boletín interno y la intranet de la empresa. 
Tabla 1. Ecosistema transmedia Play to Move

\begin{tabular}{|l|l|l|}
\hline Gamificación & Viaje & Grupo de Yammer \\
\hline Juego (app) & Brand film & Píldoras audiovisuales \\
\hline Micro-retos & Acciones en el estreno del brand film & Publicaciones \\
\hline
\end{tabular}

\subsection{Historia}

A nivel narrativo la campaña propone como argumento el viaje del héroe, un patrón narrativo que se repite continuamente en las historias y leyendas de diferentes culturas y momentos de la historia, mostrando una continuidad con esa narrativa germinal (Balló; Pérez, 1997). Esta estructura mitológica o monomito, tal y como la denomina Campbell (2014), se compone de tres etapas principales -partida, iniciación y regreso-, a través de las cuales el héroe rompe con su rutina diaria para embarcarse en una aventura.

La trama de la campaña se centra en la búsqueda de la aplicación de los principios de negocio responsable de la compañía en los proyectos que ésta lleva a cabo a lo largo del mundo. La trama de la búsqueda favorece la utilización de proyectos transmedia porque fomentan la curiosidad de las audiencias y su participación en el proceso (Costa-Sánchez, 2014).

Tabla 2. Etapas de la narración transmedia de Play to Move

\begin{tabular}{|c|c|c|}
\hline Etapa de la historia & Descripción & Plataformas y formatos \\
\hline El mundo ordinario & $\begin{array}{l}\text { En la primera etapa, los empleados se encuentran en una situación de } \\
\text { normalidad, esperando a que algo suceda. Es entonces cuando de mane- } \\
\text { ra inesperada surge una oportunidad para salir de su mundo cotidiano. } \\
\text { En este caso, la compañía plantea un juego, con pequeños problemas que } \\
\text { los empleados tendrán que solventar y que permitirán que éstos adquie- } \\
\text { ran sabiduría y puedan enfrentarse a retos mayores. }\end{array}$ & $\begin{array}{l}\text { Gamificación en Yammer. } \\
\text { Grupo de dinamización en Yammer. }\end{array}$ \\
\hline La llamada a la aventura & $\begin{array}{l}\text { Fruto del juego, los tres empleados con mejores puntuaciones son } \\
\text { llamados a participar en una aventura, un viaje a tres países, en el que } \\
\text { los protagonistas se ven obligados a poner en práctica todo lo que han } \\
\text { aprendido. Tal y como se afirma en el brand film, serán los embajadores } \\
\text { de la compañía para comprobar cómo se materializan los principios de } \\
\text { negocio responsable y verificar si Telefónica realmente está contribuyen- } \\
\text { do a construir una sociedad mejor o no. }\end{array}$ & $\begin{array}{l}\text { Notificación a los empleados seleccio- } \\
\text { nados (carta). } \\
\text { Grupo de dinamización en Yammer. }\end{array}$ \\
\hline $\begin{array}{l}\text { Inicio del viaje. } \\
\text { Retos }\end{array}$ & $\begin{array}{l}\text { Los empleados abandonan su zona de confort y viajan a través de tres } \\
\text { países y varias ciudades para conocer los proyectos de la compañía. En } \\
\text { el viaje, son acompañados por varios maestros (trabajadores de la com- } \\
\text { pañía), que a través de experiencias les ayudarán a construir una nueva } \\
\text { identidad asociada al negocio responsable de la compañía. }\end{array}$ & \multirow[t]{2}{*}{$\begin{array}{l}\text { Viaje experiencial. } \\
\text { Grupo de dinamización en Yammer. }\end{array}$} \\
\hline $\begin{array}{l}\text { Renacimiento y } \\
\text { recompensa }\end{array}$ & $\begin{array}{l}\text { Tras finalizar el viaje, los protagonistas consiguen superar la prueba y } \\
\text { obtienen la recompensa, convirtiéndose en personas nuevas que ahora } \\
\text { servirán como ejemplo para otros compañeros. }\end{array}$ & \\
\hline La vuelta a casa & $\begin{array}{l}\text { De vuelta a la normalidad, los tres empleados se ven obligados a trans- } \\
\text { mitir todo ese conocimiento que han adquirido durante el viaje al resto } \\
\text { de compañeros, en este caso, a través de un brand film. De esta manera, } \\
\text { todos los empleados podrán aplicar los conocimientos y estrategias } \\
\text { aprendidas a su actividad diaria. }\end{array}$ & $\begin{array}{l}\text { Brand film. } \\
\text { Micro-retos. } \\
\text { Grupo de dinamización en Yammer. }\end{array}$ \\
\hline
\end{tabular}

Mediante la narración, Telefónica logra transformar la información y los datos de la compañía en emociones que conectan directamente con las personas, y consigue que éstas inviertan su tiempo y atención a las mismas (Núñez-López, 2007).

\subsection{El rol de los empleados}

La campaña se construye a través de una narrativa interactiva (Orihuela, 1997), es decir, a pesar de que la empresa mantiene un control sobre el contenido y la forma del relato, éste se encuentra abierto a variaciones por parte del público interno.

Encontramos diferentes niveles de participación de los públicos internos en la construcción del relato. Los empleados adquieren un rol de receptores pasivos tanto en el brand film como en otras de las acciones promocionales, ya que se limitan a recibir y consumir el contenido. En el grupo de Yammer así como en el juego, los públicos internos adquieren un rol de receptores activos, ya que de manera voluntaria deciden realizar un seguimiento activo de la campaña a través del consumo de los contenidos, demostrando su implicación. El nivel de participación más alto sería el del rol de co-creador, que adquiere su máxima expresión en el viaje experiencial y su distribución al resto de empleados a través de la creación de contenido en varios formatos, así como con los "Micro-retos Play to Move", si bien tanto en el grupo de Yammer como en el juego los públicos también pueden adoptar este nivel de participación interactuando con la historia y creando contenido. 
Durante toda la campaña se observa cómo la empresa realiza acciones para incentivar esa participación de los públicos, que deciden de manera voluntaria su grado de implicación con la historia.

\subsection{Evaluación de los resultados e impacto en la responsabilidad social de la empresa}

La implicación de los empleados, áreas y países fue alta durante toda la campaña, tal y como demuestran los datos de actividad y engagement en los medios y plataformas, lo que favoreció el alto alcance de la campaña y la relación entre los empleados participantes. La app (juego) acumuló más de 5.000 jugadores y 60.000 partidas en 15 países, mientras que el grupo de Yammer se constituyó como el más activo de Telefónica a nivel mundial, con la participación de 4.380 empleados, más de 200 publicaciones propias, 1.700 me gusta, 1.500 comentarios, 100 comparticiones y 3.800 interacciones con usuarios. La película fue visionada por más del $80 \%$ de la plantilla, y a su estreno asistieron 400 empleados, directivos y representantes.

La iniciativa contribuyó al conocimiento de los principios de negocio responsable de la compañía. Se midió el conocimiento de los principios de negocio responsable antes y después de la campaña observándose una diferencia significativa. Concretamente, el $74 \%$ de los empleados aseguró que la campaña había cambiado su percepción sobre el negocio responsable de la empresa.

\author{
La gestión de los tiempos, el carácter \\ internacional de la campaña o generar \\ contenido para diferentes plataformas, \\ son los principales retos de una campa- \\ ña transmedia de estas características
}

De la misma manera los entrevistados también confirmaron que las encuestas de clima de la compañía mostraron una mejora respecto a la percepción de Telefónica como empresa responsable entre el público interno, lo que contribuyó así mismo al sentimiento de pertenencia a la empresa.

Según los responsables de la campaña, algunas de las claves del éxito fueron su carácter novedoso y el tono informal que se utilizó durante toda la iniciativa. La gestión de los tiempos (timing), el carácter internacional de la campaña o la necesidad de generar contenido para diferentes plataformas, fueron algunos de los principales retos experimentados por los responsables a la hora de gestionar una campaña transmedia de estas características.

A pesar del éxito de la iniciativa, los responsables recuerdan que la comunicación de la responsabilidad social es algo que necesita gestionarse de manera continua.

"Refuerzas el conocimiento, pero al final tienes que estar siempre recordando las cosas, insistiendo... [...] No es "un día hago una campaña y el tema lo doy por sabido" [...] Además, los principios de negocio responsable se van concretando en diferentes políticas y normativas, por eso siempre estamos compartiendo esos nuevos contenidos, porque están vivos" (Lourdes Tejedor).

También admiten que es difícil realizar el salto entre el conocimiento de los principios de negocio responsable a su aplicación práctica en el día a día de los empleados:

“No hemos conseguido un salto definitivo. Puedes tener gente más dispuesta, más colaboradora, pero tienes que seguir insistiendo para mantener esa voluntad y ese ánimo de participación y de colaboración" (Lourdes Tejedor).

A pesar de ello, afirman que no cuentan con métricas específicas para medir esa participación del público interno en su política de negocio responsable:

"Es tan amplio el negocio responsable que no tenemos una manera de medirlo. Es difícil cubrir todo el abanico y ver la participación" (Lourdes Tejedor).

En resumen, el éxito de la campaña demuestra que la narrativa transmedia es un enfoque efectivo de relaciones públicas para comunicar la estrategia de responsabilidad social de las empresas, sin olvidar la importancia de la participación de los empleados en esas políticas responsables. De acuerdo con los responsables de la campaña, "el transmedia es el futuro" (Eduardo Puig De la Bellacasa), si bien recuerdan que requiere de bastantes recursos económicos, dada la cantidad de formatos y canales que es necesario abordar, contenidos y tiempo. También admiten que es importante jugar con el factor novedad:

"Hacer una campaña de este calado todos los años al final lo que hace es perder efectividad", afirma Puig de la Bellacasa.

\section{Discusión y conclusiones}

Las formas de comunicación entre las organizaciones y sus públicos internos se encuentran en constante evolución. Las organizaciones tienen que enfrentarse al reto de alcanzar a un público que cada vez está más expuesto a grandes cantidades de información y con unos hábitos de consumo multi-plataforma. También se trata de un público cada vez más empoderado, pudiendo adoptar al mismo tiempo el rol de emisor-receptor gracias a las posibilidades que brinda la tecnología.

Más allá de las campañas de comunicación interna convencionales que utilizaban una cantidad reducida de plataformas y formatos, fundamentalmente de carácter unidireccional (intranet, boletín interno, vídeos corporativos...), las campañas de comunicación transmedia se presentan como un nuevo escenario en el que confluyen todas esas tendencias. És- 
tas permiten conectar con los empleados mediante relatos que generan emociones y que se van construyendo en diferentes plataformas, pudiendo el propio público interno participar en la co-creación de esa historia, de manera lúdica y experiencial.
Según los responsables resulta difícil enlazar los principios de negocio responsable con su aplicación práctica en el día a día de los empleados

En este caso de estudio, Telefónica demostró cómo se pueden utilizar las narrativas transmedia para contar su estrategia de negocio responsable de manera efectiva. Cada uno de los medios y formatos utilizados cumplía una función:

- la gamificación sirvió para transmitir de una forma amena y atractiva los principios de negocio responsable e implicar a todos los empleados en la historia, generando expectación;

- el viaje y la creación del brand film fueron utilizados para la generación de la narrativa;

- I grupo de Yammer y los micro-retos se utilizaron para contar el relato de manera no secuencial, y fomentar la interacción y participación de todos los empleados.

Estos resultados se muestran en línea con estudios anteriores, en los que se concluye que los discursos de las organizaciones deben transformarse en historias, desde un acercamiento lúdico e interactivo, creadoras de experiencias (Costa-Sánchez, 2014, p. 147).

La RSE propicia un espacio de encuentro con los públicos internos y las narrativas transmedia, suponen una vía para provocar y potenciar el compromiso de los agentes interesados y convertirlos en partícipes de los relatos sobre el negocio responsable. Establecer canales de participación (mediante estas nuevas estrategias) potencia que la RSE contribuya de forma efectiva a su propia esencia, así como a dar respuesta a las demandas de los públicos por un mayor desempeño y compromiso; sobre todo cuando hablamos de grandes multinacionales y de cómo deben hacer frente y compensar los impactos que generan. En esta línea encontramos trabajos como el de Coombs $(2019$, p. 351) que subraya el potencial de la narrativa transmedia para generar un mayor compromiso y emoción del público con los mensajes de la RSE y compensar así el rechazo que genera la publicidad ligada a la RSE.

A pesar de sus posibilidades, las narrativas transmedia han sido muy poco aplicadas a la comunicación interna en el caso de España hasta el momento. De hecho, no se tiene constancia de ningún precedente más allá del estudio de caso del presente artículo, si bien su importancia comienza a ser reconocida al menos en el ámbito profesional (Cornejo, 2019). Las organizaciones se encuentran ante el reto de evolucionar hacia estrategias narrativas, que incluyan la participación de sus públicos y que permitan ser consumidas a través de diferentes entradas, digitales y no digitales. El futuro de las relaciones públicas y de la comunicación interna pasa por la confluencia con otras disciplinas como la publicidad o el entretenimiento, y la incorporación de la gamificación, el storytelling y la transmedialidad.

\section{Referencias}

Balló, Jordi; Pérez, Xavier (1997). La semilla inmortal: los argumentos universales en el cine. Barcelona: Anagrama. ISBN: 9788433905482

Briceño, Sonia; Mejías, Iraida; Moreno, Fidel (2010). “La comunicación corporativa y la responsabilidad social empresarial (RSE)". Daena: International journal of good conscience, v. 5, n. 1, pp. 37-46.

http://www.spentamexico.org/v5-n1/5(1)37-46.pdf

Campbell, Joseph (2014). El héroe de las mil caras. Psicoanálisis del mito. México: Fondo de Cultura Económica. ISBN: 9786071620132

Capriotti, Paul; Moreno, Ángeles (2007). "Communicating corporate responsibility through corporate web sites in Spain". Corporate communications: An international journal, v. 12, n. 3, pp. 221-237.

https://doi.org/10.1108/13563280710776833

Castaño-González, Esther-Julia (2011). “Comunicar la responsabilidad social, una opción de éxito empresarial poco explorada". Revista lasallista de investigación, v. 8, n. 2, pp. 173-186.

https://www.redalyc.org/articulo.oa?id=69522607019

Castillo-Esparcia, Antonio (2009). Relaciones públicas. Teoría e historia. Barcelona: Editorial UOC. ISBN: 9788497888172

Chomón-Serna, José-María; Busto-Salinas, Lorena (2018). “Ciencia y transmedia: binomio para la divulgación científica. El caso de Atapuerca". El profesional de la información, v. 27, n. 4, pp. 938-946.

https://doi.org/10.3145/epi.2018.jul.22

Coombs, Timothy (2019). "Transmedia storytelling: A potentially vital resource for CSR communication". Corporate communications: An international journal, v. 24, n. 2, pp. 351-367.

https://doi.org/10.1108/CCIJ-11-2017-0114

Cornejo, David (2019). Comunicación interna transmedia. Guía en 12 pasos para empresas y organizaciones. Santiago de Chile. ISBN: 9789563984026

https://es.slideshare.net/DavidCornejo6/comunicacion-interna-transmedia 
Corona-Rodríguez, José-Manuel (2015). Educación transmedial: estrategias y desafíos para investigar el aprendizaje informal en el escenario hipermediático.

http://reposital.cuaed.unam.mx:8080/xmlui/handle/20.500.12579/4531

Corporate Excellence (2018). Play to Move. Viviendo los principios de negocio responsable de Telefónica. Corporate Excellence.

https://uploads.strikinglycdn.com/files/5f954fb3-7260-45bd-a0b6-94720b4f4ff9/Caso\%20Telef\%C3\%B3nica\%20rev-4.pdf

Costa-Sánchez, Carmen (2013). “Narrativas transmedia nativas: ventajas, elementos de la planificación de un proyecto audiovisual transmedia y estudio de caso". Historia y comunicación social, v. 18, pp. 561-574.

https://doi.org/10.5209/rev_HICS.2013.v18.44349

Costa-Sánchez, Carmen (2014). "Transmedia storytelling, an ally of corporate communication: \#Dropped by Heineken case study". Comunicación \& society, v. 27, n. 3, pp. 127-150.

https://www.unav.edu/publicaciones/revistas/index.php/communication-and-society/article/view/35992

Costa-Sánchez, Carmen; Piñeiro-Otero, Teresa (2012). “Nuevas narrativas audiovisuales: multiplataforma, crossmedia y transmedia. El caso de Águila Roja (RTVE)”. Icono14, v. 10, n. 2, pp. 102-125.

https://doi.org/10.7195/ri14.v10i2.156

Cutlip, Scott M.; Center, Allen H. (2000). Relaciones públicas eficaces. Clásicos de las relaciones públicas. Barcelona: Gestión 2000. ISBN: 8480885734

Davidson, Drew (2008). Stories in between: Narratives and mediums @ play. Lulu.com. ISBN: 9781435720800 https://pdfs.semanticscholar.org/15b0/a538bcf1217dfea5027855da26bd9c4fe9c1.pdf

Ethos (2017). Indicadores Ethos para negocios sustentáveis e responsáveis. Glossário. Instituto Ethos de Empresas y Responsabilidad Social.

https://www3.ethos.org.br/wp-content/uploads/2017/06/Gloss\%C3\%A1rio-2017.pdf

Ferrari, María-Aparecida; Durán, Ana-María (2018). “El estado del arte de las relaciones públicas y la sustentabilidad en organizaciones brasileñas y ecuatorianas". Revista internacional de relaciones públicas, v. 8, n. 16, pp. 129-150. https://doi.org/10.5783/RIRP-16-2018-08-129-150

Ferré-Pavia, Carme; Hiyo-Tambra, Cristie (2018). "La información accesible sobre RSE en Sudamérica en los campos de la universidad, la administración y las empresas. Estudio exploratorio y comparativo de 10 empresas". Revista universidad y empresa, v. 20, n. 35, pp. 221-249.

https://doi.org/10.12804/revistas.urosario.edu.co/empresa/a.6028

Fuentes-Gómez-Calcerrada, Juan-Luis; González-Martín, María-del-Rosario (2015). “Emprendimiento y responsabilidad social. La estrategia educativa de las narrativas 'transmedia'”. En: Núñez-Cubero, Luis (coord.). Cultura emprendedora y educación. Sevilla: Universidad de Sevilla, pp. 233-240. ISBN: 9788447216062

Galán, Esteban (2016). "Relato transmedia vinculado a marcas: el personaje de Benito como instrumento de branded content". El profesional de la información, v. 25, n. 6, pp. 915-922.

https://doi.org/10.3145/epi.2016.nov.08

Galbiati, Marisa; Venditti, Simona (2017). “Communication design for social impact. Using digital media and storytelling practices for disseminating CSR activities". En: IX Congreso intl de diseño de La Habana. Forma 2017. https://re.public.polimi.it/retrieve/handle/11311/1029253/218225/pdf_CSR_formahabana.pdf

Grandío-Pérez, María-del-Mar (2016). “El transmedia en la enseñanza universitaria. Análisis de las asignaturas de educación mediática en España (2012-2013)”. Palabra clave, v. 19, n. 1, pp. 85-104.

https://doi.org/10.5294/pacla.2016.19.1.4

Jenkins, Henry (2009). "The revenge of the origami unicorn: Seven principles of transmedia storytelling (well, two actuaIly. Five more on Friday)". Confessions of an aca-fan. The official weblog of Henry Jenkins, December 12.

http://henryjenkins.org/2009/12/the_revenge_of_the_origami_uni.html

Jenkins, Henry; Clinton, Katie; Purushotma, Ravi; Robison, Alice J.; Weigel, Margaret (2006). Confronting the challenges of participatory culture: Media education for the $21^{\text {st }}$ century. The MacArthur Foundation.

https://www.macfound.org/media/article_pdfs/JENKINS_WHITE_PAPER.PDF

Kim, Hyo-Sook (2011). "A reputational approach examining publics attributions on corporate social responsibility motives". Asian journal of communication, v. 21, n. 1, pp. 84-101.

https://doi.org/10.1080/01292986.2010.524230

La-Roda-Gómez, Aída (2017). La comunicación interna de la calidad en la universidad española, propuesta de un modelo teórico transmedia. Tesis doctoral. Valencia: Universidad CEU-Cardenal Herrera.

https://repositorioinstitucional.ceu.es/handle/10637/8532 
Llorente \& Cuenca (2016). Informe anual 2016.

https://informeanual2016./lorenteycuenca.com/los-clientes-en-el-centro-del-negocio

Llorente \& Cuenca (2017). "La primera campaña transmedia de comunicación interna de una empresa española". TOP comunicación \& RRPP.

http://www.topcomunicacion.com/noticia/8860/primera-campana-transmedia-de-comunicacion-interna-tlelefonicaempresa-espanola

López-Castillo, Yosue-Shalom (2017). “Chipotle responsabilidad social y transmedia”, Bitácora del Kapitán, 31 mayo. https://comunidad.iebschool.com/bitacoradelkapitan/2017/05/31/chipotle-responsabilidad-social-y-transmedia

Maltseva, Kateryna; Fieseler, Christian; Trittin-Ulbrich, Hannah (2019). "The challenges of gamifying CSR communication". Corporate communications: An international journal, v. 24, n. 1, pp. 44-62.

https://doi.org/10.1108/CCIJ-09-2018-0092

Mayor-Mayor, Francesc (2014). "Transmedia storytelling desde la ficción televisiva serial española: El caso de Antena 3". CIC. Cuadernos de información y comunicación, v. 19, pp. 69-85.

https://www.redalyc.org/articulo.oa?id=93530573005

Míguez-González, María-Isabel (2011). "La investigación sobre relaciones públicas en las revistas españolas de comunicación (2008-2010)”. En: Fernández-Souto, Ana-Belén; García-García, Francisco (eds.). VI Congreso internacional de investigación y relaciones públicas, pp. 124-138. Asociación Científica de Investigación de las Nuevas tecnologías de la Comunicación Icono14. ISBN: 9788493907716

http://airrpp.org/wp-content/uploads/2012/12/actas_VIcongreso_AIRP.pdf

Nicoli, Nicholas; Komodromos, Marcos (2019). "CSR communication in the digital age. The case of The Bank of Cyprus". In: Antonaras, Alexandros; Dekoulou, Paraskevi (eds.). Cases on corporate social responsibility and contemporary issues in organizations. Pennsylvania: IGI Global, pp. 71-89. ISBN: 9781522577157

https://doi.org/10.4018/978-1-5225-7715-7.ch005

Niño-Benavides, Teresa-del-Pilar; Cortés-Cortés, María-Isabel (2018). “Comunicación estratégica y responsabilidad social empresarial, escenarios y potencialidades en creación de capital social: una revisión de la bibliografía". Prisma social, n. 22, pp. 127-158.

http://revistaprismasocial.es/article/view/2570/2756

Núñez-López, Antonio (2007). iSerá mejor que lo cuentes!: Los relatos como herramientas de comunicación (storyteIling). Barcelona: Empresa Activa. ISBN: 9788496627307

Orihuela, José-Luis (1997). "Narraciones interactivas: el futuro no lineal de los relatos en la era digital". Palabra-clave, n. 2, pp. 37-47.

http://palabraclave.unisabana.edu.co/index.php/palabraclave/article/viewFile/338/480

Orozco-Toro, Jaime-Alberto; Ferré-Pavia, Carme (2013). “La comunicación estratégica de la responsabilidad social corporativa". Razón y palabra, n. 83, pp. 706-726.

http://www.razonypalabra.org.mx/N/N83/V83/20_OrozcoFerre_V83.pdf

Orozco-Toro, Jaime-Alberto; Ferré-Pavia, Carme (2017). “La percepción de la reputación corporativa de los stakeholders de una empresa de comunicación: cómo afecta a Televisió de Catalunya comunicar una acción de responsabilidad social corporativa". Palabra clave, v. 20, n. 2, pp. 473-505.

https://doi.org/10.5294/pacla.2017.20.2.8

Patton, Michael-Quinn (1990). Qualitative research and evaluation methods. Thousand Oaks, CA: Sage. ISBN: 0803937792

Robin, Bernard (2005). "The educational uses of digital storytelling". In: Crawford, Caroline; Carlsen, Roger; McFerrin, Karen; Price, Jerry; Weber, Roberta; Willis, Dee-Anna (eds.). Proceedings of SITE 2006--Society for information technology \& teacher education int conf. Florida, USA: Association for the Advancement of Computing in Education (AACE), pp. 709-716 https://www.learntechlib.org/primary/p/22129

Rodríguez-Ruiz, Jaime-Alejandro; López-Peinado, Laura D.; González-Gutiérrez, Luis-Felipe (2015). "La narrativa transmedia como experiencia de simulación de inteligencia colectiva. El caso de Atrapados". Signo y pensamiento, v. 34, n. 67, pp. 60-74.

https://doi.org/10.11144/Javeriana.syp34-67.ntes

Rodríguez-Terceño, José (2014). “Cine y nuevas fórmulas comunicativas: evolución del emplazamiento publicitario hecho cine". En: Requeijo-Rey, Paula; Gaona-Pisonero, Carmen (coords.). Contenidos innovadores en la universidad actual. Madrid: McGraw-Hill. ISBN: 97884 48197445, pp. 611-622.

Rodríguez-Terceño, José; Caldevilla-Domínguez, David; Ramos-Fernández, Luis-Fernando (2015). “'Dramanagement': cine, empresa y responsabilidad social corporativa". Opción, v. 31, n. 1, pp. 23-40.

https://www.redalyc.org/articulo.oa?id=31043005002 
Ruiz-Muñoz, María-Jesús; Ruiz-Mora, Isabel (2018). “La exploración de metodologías docentes en el contexto de la transcomunicación”. En: XIV Congreso de la Asociación Latinoamericana de Investigadores de la Comunicación (Alaic), Comunicación en sociedades diversas: Horizontes de inclusión, equidad y democracia (no publicado).

https://riuma.uma.es/xmlui/handle/10630/16535

Saavedra-Bautista, Claudia; Cuervo-Gómez, William-Oswaldo; Mejía-Ortega, Iván-Darío (2017). “Producción de contenidos transmedia, una estrategia innovadora". Revista científica, v. 28, n. 1, pp. 6-16.

http://www.scielo.org.co/pdf/cient/n28/2344-8350-cient-28-00006.pdf

https://doi.org/10.14483/udistrital.jour.RC.2017.28.a1

Sáenz-Acosta, César; Ventura-Egoávil, José (2014). "El retorno social de la inversión: ¿'se puede medir el impacto de la difusión de un proyecto de responsabilidad social empresarial?". Revista universidad y empresa, v. 16, n. 26, pp. 31-64. https://doi.org/10.12804/rev.univ.empresa

Salmon, Christian (2008). Storytelling. La máquina de fabricar historias y formatear las mentes. Barcelona: Quinteto. ISBN: 9788483078358

Scolari, Carlos A. (2013). Narrativas transmedia. Cuando todos los medios cuentan. Barcelona: Deusto. ISBN: 9788423413362

Scolari, Carlos A. (2014). "Narrativas transmedia: nuevas formas de comunicar en la era digital". Anuario AC/E de cultura digital, pp. 71-81.

https://www.accioncultural.es/media/Default\%20Files/activ/2014/Adj/Anuario_ACE_2014/6Transmedia_CScolari.pdf

Scolari, Carlos A. (2016). "Alfabetismo transmedia: estrategias de aprendizaje informal y competencias mediáticas en la nueva ecología de la comunicación”. Telos: Cuadernos de comunicación e innovación, n. 103, pp. 13-23.

https://repositori.upf.edu/handle/10230/27788

Scolari, Carlos A.; Masanet, Maria-José; Guerrero-Pico, Mar; Establés, María-José (2018). "Transmedia literacy in the new media ecology: Teens' transmedia skills and informal learning strategies". El profesional de la información, v. 27, n. 4, pp. 801-812.

https://doi.org/10.3145/epi.2018.jul.09

Seitel, Fraser P. (2002). Teoría y práctica de las relaciones públicas. Madrid: Prentice Hall. ISBN: 9788420535104

Sugathan, Savita K.; Kalid, Khairul-Shafee (2009). "An exploratory study of storytelling approach as an instructional tool from educators' perspective". In: Icctd 2009. 2009 Intl conf on computer technology and development, pp. $480-483$.

https://doi.org/10.1109/ICCTD.2009.122

Telefónica (2016a). Informe integrado 2016.

https://www.telefonica.com/es/web/negocio-responsable/informe-2016

Telefónica (2016b). Play to Move, La película, Brand film (48:28 min).

https://www.youtube.com/watch?v=AzjcRYogH8s

Torres-Valdés, Rosa-María; Campillo-Alhama, Concepción (2013). “Desarrollo local y relaciones públicas para grupos desfavorecidos en la Comunidad de Madrid". Prisma social, n. 10, pp. 394-432.

http://www.isdfundacion.org/publicaciones/revista/numeros/10/secciones/abierta/02-desarrollo-local.html

Tsou, Wenli; Wang, Weichung; Tzeng, Yenjun (2003). "Applying computer multimedia storytelling website in foreign language learning". In: Procs $3^{\text {rd }}$ IEEE Intl conf on advanced learning technologies, pp. 262-263.

http://doi.org/10.1109/ICALT.2003.1215074

Villén-Higueras, Sergio-Jesús (2017). “Estrategias transmedia en las series de televisión: la transformación de una tie-in website en el inicio de un ARG (caso Discover Westworld)". AdComunica, n. 14, pp. 119-140.

http://www.adcomunicarevista.com/ojs/index.php/adcomunica/article/view/395/362

Wang, Jian; Chaudhri, Vidhi (2009). “Corporate social responsibility engagement and communication by Chinese companies". Public relations review, v. 35, n. 3, pp. 247-250.

https://doi.org/10.1016/j.pubrev.2009.04.005

Wilcox, Dennis L.; Cameron, Glen T.; Xifra, Jordi (2012). Relaciones públicas: estrategias y tácticas. 10ª ed. Madrid: Pearson Educación. ISBN: 9788483228135

Würfel, Maren (2014). "Transmedia appropriation and socialization processes among German adolescents". International journal of communication, n. 8, pp. 2240-2258.

https://ijoc.org/index.php/ijoc/article/view/2698/1206

Xifra, Jordi; Chias, Josep (2008). El marqueting. Las relaciones públicas. Barcelona: Editorial UOC. ISBN: 9788497887144

Yin, Robert K. (2013). Case study research: Design and methods. Newbury Park CA: Sage. ISBN: 9781452242569 